\title{
Clinical Effect of Revascularization Strategies and Pharmacologic Treatment on Long-Term Results in Patients with Advanced Peripheral Artery Disease with TASC C and D Femoropopliteal Lesions
}

\author{
Chiu-Yang Lee $\mathbb{D}^{1,2,3}$ \\ ${ }^{1}$ Division of Cardiovascular Surgery, Department of Surgery, Taipei Veterans General Hospital, Taipei, Taiwan \\ ${ }^{2}$ Institute of Clinical Medicine, National Yang Ming Chiao Tung University, Taipei, Taiwan \\ ${ }^{3}$ National Defense Medical Center, Taipei City, Taiwan \\ Correspondence should be addressed to Chiu-Yang Lee; david.lee0501@gmail.com
}

Received 12 August 2021; Revised 25 November 2021; Accepted 13 December 2021; Published 4 March 2022

Academic Editor: Piotr Musialek

Copyright (c) 2022 Chiu-Yang Lee. This is an open access article distributed under the Creative Commons Attribution License, which permits unrestricted use, distribution, and reproduction in any medium, provided the original work is properly cited.

Background. This study was to assess the clinical outcome and associated parameters of endovascular therapy (EVT group) and bypass surgery (bypass group) in patients with long femoropopliteal TransAtlantic Inter-Society Consensus II (TASC II) C and D peripheral artery disease (PAD). Methods. 187 patients who underwent successful EVT or bypass surgery were assessed. The endpoints included the events of cardiovascular disease (CVD) and lower-extremity amputation (LEA), 3-year primary patency, and 3-year amputation-free survival (AFS). Results. The 3-year primary and secondary patency rates were better in the bypass group ( $P=0.007$ and $P=0.039$, respectively), while the incidences of LEA, new CVD events, and mortality were comparable between groups. Weighted multivariate Cox analyses showed that cilostazol treatment (hazard ratio (HR): $0.46,95 \%$ confidence interval (CI): $0.3-0.72, P=0.001$ ), statin treatment (HR: 0.54, 95\% CI: 0.33-0.9, $P=0.014$ ), and direct revascularization (DR) (HR: $0.47,95 \%$ CI: $0.29-0.74, P=0.001$ ) were predictive factors of 3-year primary patency. Kaplan-Meier curve analyses of timeto-primary cumulative AFS showed that nondiabetes mellitus, mild PAD, and cilostazol and statin treatment were correlated with a superior 3-year AFS (log rank test, $P=0.001, P<0.001, P=0.009$, and $P=0.044$, respectively). Conclusions. Endovascular stenting based on the angiosome concept and bypass surgery provide comparable benefits for the treatment of long, advanced femoropopliteal lesions after a short follow-up period, whereas cilostazol therapy for more than 3 months, aggressive treatment of dyslipidemia, and surgical revascularization were associated with higher primary patency.

\section{Introduction}

Peripheral arterial disease (PAD) is a prevalent type of atherosclerosis and is similar to coronary artery disease (CAD), which is also caused by atherosclerosis.

Critical limb ischemia (CLI) is associated with high cardiovascular disease mortality and lower-extremity amputation (LEA) $[1,2]$; it is the most severe form of PAD, presenting as gangrene, pain at rest, and ischemic ulcer necrosis. The TransAtlantic Inter-Society Consensus (TASC) II guidelines recommend revascularization approaches including endovascular intervention and bypass surgery for CLI. Recent series of studies have shown that $10-20 \%$ of lower-limb revascularization procedures are performed surgically, and approximately $80 \%$ are endovascular. The TASC II guidelines recommend endovascular intervention as the optimal option for the treatment of CLI to relieve pain, assist wound healing, prevent limb loss, and improve patient function and quality of life.

Despite the advantages of endovascular intervention for CLI management, including a lower surgical risk and lower mortality, mean patency rates of $56 \%$ to $77 \%$ at 1 year and $39 \%$ to $80 \%$ at 5 years have been reported, depending on the revascularization method and location of the disease [3-5]. Taking long-term durability into consideration, bypass surgery remains the optimal treatment for multilevel and 
long femoropopliteal lesions not subject to endovascular intervention and provides adequate arterial perfusion to the foot, resulting in an elevated limb salvage rate and long-term durability $[6,7]$. The aforementioned studies demonstrated that in the first year, approximately $25 \%$ of these patients experienced resolution of their symptoms, while $20 \%$ continued to have symptoms, $30 \%$ underwent amputation, and $25 \%$ expired. Taking the results of all of the aforementioned reviews together, the unique emphasis on surgical or endovascular revascularization strategies as the basis of current treatment for long femoropopliteal lesions in patients with PAD was not sufficient without comprehensive strategies for reduction of restenosis and arterial remolding.

To our knowledge, evidence regarding the presence of cardiovascular risk factors, morbidity, and mortality associated with revascularization methods has been presented, but data regarding the effects of individual predisposing factors and pharmacotherapy on the primary patency of target lesion, clinical outcomes, and limb function are conflicting. The aim of the study was to investigate the clinical outcome and associated parameters in patients with CLI receiving endovascular therapy and bypass surgery.

\section{Materials and Methods}

2.1. Study Population and Design. The study was conducted in accordance with the Declaration of Helsinki. Prior to the study, the protocol was approved by the Ethics Committee of Taipei Veterans General Hospital (No. 2015-03-016AC). All patients gave written informed consent before revascularization. This study retrospectively analyzed our prospectively maintained institutional database, which included 595 consecutive advanced PAD patients (Rutherford classification III, IV, V, and VI) who underwent bypass surgery or endovascular therapy (EVT, angioplasty plus bare metal stent (BMS) or covered stent (Viabahn)) in a single medical center from February 2009 to March 2015. Of these, 187 patients with 229 limbs satisfied the inclusion criteria discussed further.

The inclusion criteria were patients between the ages of 20 and 90 years; with a first diagnosis of PAD; severe PAD (Rutherford classification III, IV, and V) manifested as severe claudication, rest pain, or ischemic tissue loss; ischemic tissue loss associated with an ankle pressure $<70 \mathrm{mmHg}$ or a toe pressure $<50 \mathrm{mmHg}$; long femoropopliteal TASC C and D lesions; successful bypass surgery or endovascular therapy (stenting), obtaining flow through at least one vessel to the pedal arch; treatment with cilostazol; and fair compliance and regular monitoring during follow-up. The exclusion criteria included acute artery embolism; Buerger's disease; prior amputation (minor or major); congestive heart failure (CHF) according to New York Heart Association (NYHA) function III-IV or an ejection fraction of $<35 \%$; bleeding diathesis, acute cardiovascular diseases, or acute cerebrovascular diseases; and active diseases such as hepatitis, malignancy, or systemic infection. These patients were excluded before intervention.

According to the angiosome-oriented revascularization strategy, endovascular and surgical revascularization were applied in this study. If patients had plantar ulcers, we made an effort to treat the posterior tibial artery first. If this artery was not revascularized, we then treated the anterior tibial artery. Direct revascularization (DR) provides adequate blood flow directly to the ischemic tissue area over the direct angiosome, while indirect revascularization (IR) provides blood flow to the ischemic limb over the indirect angiosome through the collateral vessels [8-10].

In total, 187 patients with advanced PAD mainly involving long femoropopliteal lesions (TASC II C and D) were included in this study. The patients were divided into bypass (bypass surgery) and EVT groups.

2.2. Medication. Patients undergoing stenting were given dual antiplatelet therapy for at least 3 months followed by single antiplatelet therapy. For patients undergoing bypass surgery, single antiplatelet therapy was prescribed. All patients in this study were treated with cilostazol (50-100 mg bid) after the intervention. Patients who received continuous cilostazol therapy for at least 3 months after surgery were considered to have received long-term cilostazol therapy, while those who received intermittent treatment or treatment for fewer than 3 months were defined as receiving short-term treatment. The duration of cilostazol treatment depended on resolution of the claudication and leg pain, and relief of the clinical condition. In addition, fewer than half of the patients were treated with statins to control total cholesterol and LDL levels to $200 \mathrm{mg} / \mathrm{dl}$ and $130 \mathrm{mg} / \mathrm{dl}$, respectively.

2.3. Study Endpoints. All patients underwent clinical, ABI, and Doppler ultrasound examinations every three to six months. CTA or invasive angiography was performed when abnormal findings occurred. The primary endpoints were the primary patency rate of endovascular intervention or graft bypass of femoropopliteal lesions at $6,12,24$, and 36 months; and the secondary patency rate without extra endovascular stenting or graft bypass for target lesions at 12, 24, and 36 months. In this study, LEA was defined as a mild amputation (below the ankle) or a major amputation (above the ankle). The secondary endpoints were amputation-free survival (AFS), overall survival (OS), or overall mortality associated with a cerebrovascular accident (CVA) or sepsis at 24 months, and CAD at 12, 24, and 36 months. AFS in this study was defined as the avoidance of major amputations.

Successful revascularization was defined as residual stenosis of less than $30 \%$ upon angiography, with an indication of a minimum of one BTK artery or good distal collateralization to the plantar arch, or a postprocedural increase in the ABI by 0.10 or in ankle pressure as compared with baseline measurements.

A major adverse limb event (MALE) based on the Society for Vascular Surgery (SVS) document is a fundamental outcome measure for comparison of revascularization approaches, including target lesion reintervention (thrombectomy/thrombolysis or major surgical revision of an existing bypass or new stenting/open bypass graft) in addition to major amputation. 
Target lesion reintervention encompasses endovascular or graft bypass procedures for occlusive lesions, performed in patients with recurrent symptoms accompanied by a decrease in the ABI by 0.10 during follow-up or a return to the prior condition and recurrent stenosis $<50 \%$ as measured by duplex ultrasound imaging or CTA, or invasive angiography results showing a stenosis diameter of $\leq 50 \%$ or a stenosis area of $\leq 70 \%[11,12]$.

2.4. Statistical Analysis. The Kolmogorov-Smirnov test was employed to examine the normality of numerical variable distributions. According to the distributed results, continuous data are presented as the mean \pm standard deviation $(\mathrm{SD})$, and percentages and numbers are used to express categorical data. Frequencies and categorical variables were compared between groups using the $\chi^{2}$ test.

For numerical variables, the Mann-Whitney $U$ test and the independent sample $t$ test were used to determine intergroup differences. Patients were undergoing follow-up when any of the following conditions occurred: death, major amputation, or patency failure for cumulative patency. The Cox regression method was used to identify susceptibility factors for primary patency for 36 months. Additionally, these significant and potential factors with $P$ values $<0.15$ were analyzed using a weighted multivariate Cox regression model to determine their associations with primary patency based on the average treatment effect (ATE) $[13,14]$. Logistic regression was used to assess inverse probability weighting propensity scores (PSs) based on the severity and location of the disease. Inverse probability weighting PSs were determined according to disease severity and location using logistic regression to measure the ATE or the average treatment effect on the treated (ATT). A 2-tailed $P<0.05$ was deemed significant. All analyses were performed using SPSS version 22.0 (SPSS Inc., Chicago, IL, USA).

\section{Results}

3.1. Baseline Characteristics. From February 2009 to March 2015, a total of 187 subjects were enrolled in this study.

The EVT group included 94 patients (26 females, $27.7 \%$; $75.8 \pm 13.4$ years of age), while the bypass group included 93 patients ( 26 females, $28 \% ; 74.4 \pm 10.3$ years of age).

The patients in the two groups were well-matched in terms of the clinical characteristics and baseline demographics associated with bypass surgery and endovascular revascularization, as shown in Table 1. All patients had advanced PAD manifested as severe claudication (9.1\%), resting ischemic pain (25.7\%), or tissue loss (74.3\%) (Rutherford category $\geq \mathrm{V}$ ).

A summary of the TASC lesions, distribution of diseased vessels, and interventional characteristics is presented in Table 2. All diseased arteries were characterized using pretreatment imaging results based on the stratification of lesions as per the updated 2015 TASC II classification for aortoiliac, femoropopliteal, and infrapopliteal lesions [3]. As presented in Table 2, patients in the bypass and EVT groups had multiple lesions, and the bypass group had more advanced femoropopliteal lesions (TASC D) $(<0.001)$. All lesions were greater than $15 \mathrm{~cm}$ in both groups, and the patients in the bypass group had significantly longer femoropopliteal lesions as compared with the EVT group $(18.5 \pm 3.6 \mathrm{~cm}$ vs. $23.4 \pm 5.2 \mathrm{~cm}, P<0.001)$. All patients had at least one patent distal runoff of the foot, and there were no significant differences in the distal runoff vessels between the bypass and EVT groups $(1.96 \pm 0.79,1.78 \pm 0.72, P=0.146)$. The patients in the EVT group required BTK intervention to create better distal perfusion to the foot without the use of stents or drug-coating balloons (DCB); 29 patients (31.2\%) in the bypass group required femorodistal bypass to the BTK artery.

3.2. Endpoints. The associations of treatment methods with study endpoints at 36 months for all participants and subgroups are summarized in Table 3. In this study, 94 (100\%) patients in the EVT group received balloon angioplasty plus bare metal stent (BMS) or covered stent (Viabahn) implantation, and $93(100 \%)$ patients in the bypass group underwent bypass revascularization.

All enrolled patients experienced successful surgery, and no surgical mortality or major complications such as massive hematoma, retroperitoneal bleeding, or pseudoaneurysm occurred in either group.

Both groups had comparable $\mathrm{ABI}$ values before the intervention and one month after $(P=0.473$ and $P=0.113$, respectively), but the bypass group had a significantly higher $\mathrm{ABI}$ value at the 3 -year follow-up point $(0.53 \pm 0.16$ vs. $0.72 \pm 0.14, P<0.001$ ).

The two groups had comparable percentages of patients undergoing DR $(P=0.512)$. The 1 - and 2-year primary patency rates of the groups were comparable, whereas the bypass group had a greater primary patency rate at 3 years as compared with the EVT group ( $65.6 \%$ vs. $42.6 \%, P=0.001$ ). The secondary patency rate was similar in both groups at 24 months, and the bypass group had a better secondary patency at 3 years $(P=0.046)$. The Kaplan-Meier curves employed to analyze time-to-primary cumulative patency and AFS at 3 years are presented in Figures 1 and 2. The 3year primary patency results demonstrated the following: (A) patients who received bypass surgery had a better patency rate than patients who received stenting (log rank test, $P=0.007$ ); (B) patients treated with cilostazol for more than 3 months had a better survival rate than those treated for less than 3 months (log rank test, $P<0.001$ ); and $(C)$ survival rates of patients treated with statins were better than those of patients who did not receive statins ( $\log$ rank test, $P<0.001$ ) (Figure 1).

The 3-year AFS results showed the following: (A) patients who received cilostazol treatment for more than 3 months had a better 3-year AFS than patients who received treatment for fewer than 3 months (log rank test, $P=0.001$ ); (B) patients who received statin treatment had a superior 3year AFS than those who did not receive treatment (log rank test, $P<0.001$ ); (C) patients presented with Rutherford class $\mathrm{V}$ plus VI had an inferior 3-year AFS than patients with Rutherford class III plus IV (log rank test, $P=0.009$ ); and 
TABLE 1: Baseline demographics and characteristics associated with intervention.

\begin{tabular}{|c|c|c|c|c|}
\hline Characteristic & Total population $(n=187)$ & EVT group $(n=94)$ & Bypass group $(n=93)$ & $P$ value \\
\hline Age (years) & $75.1 \pm 11.9$ & $75.8 \pm 13.4$ & $74.4 \pm 10.3$ & 0.424 \\
\hline BMI & $23.4 \pm 4.4$ & $24 \pm 3.7$ & $22.8 \pm 5.0$ & 0.079 \\
\hline SBP & $137.1 \pm 24.1$ & $137.1 \pm 24.1$ & $137.1 \pm 24.2$ & 0.988 \\
\hline Gender (female) & $52(27.8)$ & $26(27.7)$ & $26(28.0)$ & 0.964 \\
\hline Lesion site (left) & $91(48.7)$ & 45 (47.9) & $46(49.5)$ & 0.828 \\
\hline Baseline CAD & $71(38)$ & $34(36.2)$ & $37(39.8)$ & 0.611 \\
\hline ESRD (regular dialysis) & $66(35.6)$ & $36(38.3)$ & $30(32.3)$ & 0.388 \\
\hline $\mathrm{DM}$ & $112(59.9)$ & $61(64.9)$ & $51(54.8)$ & 0.161 \\
\hline Hypertension & $154(82.4)$ & $76(80.9)$ & $78(83.9)$ & 0.588 \\
\hline Hyperlipidemia & $51(27.3)$ & $25(26.6)$ & $26(28.0)$ & 0.321 \\
\hline Baseline CVA & $24(12.8)$ & $15(16)$ & $9(9.7)$ & 0.199 \\
\hline Smoking & $75(40.3)$ & $35(37.6)$ & $40(43.0)$ & 0.455 \\
\hline Cellulitis on presentation & $145(77.5)$ & $74(78.7)$ & $71(76.3)$ & 0.697 \\
\hline Atrial fibrillation & $14(7.5)$ & $9(9.6)$ & $5(5.4)$ & 0.275 \\
\hline Baseline ABI & $0.52 \pm 0.13$ & $0.51 \pm 0.11$ & $0.53 \pm 0.16$ & 0.473 \\
\hline Calcification & $75(43.6)$ & $36(42.4)$ & $39(44.8)$ & 0.744 \\
\hline Retinopathy & $28(15)$ & $18(19.1)$ & $10(10.8)$ & 0.108 \\
\hline Neuropathy & $49(26.2)$ & $29(30.9)$ & $20(21.5)$ & 0.146 \\
\hline Rutherford classification & & & & 0.757 \\
\hline Stage III & $17(9.1)$ & $10(10.6)$ & $7(7.5)$ & \\
\hline Stage IV & $41(21.9)$ & $20(21.3)$ & $21(22.6)$ & \\
\hline Stage V plus VI & $129(69)$ & $64(68.1)$ & $65(66.9)$ & \\
\hline hsCRP (mg/L) & $4.85 \pm 5.23$ & $4.45 \pm 4.99$ & $5.28 \pm 5.49$ & 0.297 \\
\hline HAlc $(\%)$ & $7.51 \pm 1.57$ & $7.57 \pm 1.57$ & $7.43 \pm 1.59$ & 0.659 \\
\hline \multicolumn{5}{|l|}{ Concomitant medications } \\
\hline OHA alone & $65(37.8)$ & $27(31.8)$ & $38(43.7)$ & 0.107 \\
\hline Insulin alone & $55(29.4)$ & $30(31.9)$ & $25(26.9)$ & 0.450 \\
\hline
\end{tabular}

TABLE 2: Summary of TASC lesions, distribution of diseased vessels, and interventional characteristics.

\begin{tabular}{lcc}
\hline TASC II classification & EVT group $(n=94)$ & Bypass group $(n=93)$ \\
\hline Aortoiliac lesions & & $15(16.3)$ \\
A & $16(17.0)$ & $21(22.8)$ \\
B & $11(11.7)$ & $11(12.0)$ \\
C & $13(13.8)$ & $16(17.4)$ \\
D & $13(13.8)$ & \\
Femoropopliteal lesions & & $29(31.2)$ \\
C & $60(63.8)$ & $64(68.8)$ \\
D & $34(36.2)$ & \\
Infrapopliteal lesions & & $42(45.2)$ \\
B & $40(42.6)$ & $30(32.3)$ \\
C & $40(42.6)$ & $21(22.6)$ \\
D & $14(14.9)$ & $23.4 \pm 5.2(15-33.5)$ \\
Lesion length (cm) & $18.5 \pm 3.6(15-29)$ & $1.96 \pm 0.79$ \\
Number of distal runoffs & $1.78 \pm 0.72$ & $31(33.3)$ \\
1 vessel & $38(40.4)$ & $35(37.6)$ \\
2 vessels & $40(42.6)$ & $27(29.0)$ \\
3 vessels & $16(17.0)$ & 0 \\
Stent implantation & $94(100)$ & 0 \\
BTK intervention & $94(100)$ & $93(100)$ \\
Bypass surgery & 0 & $41(44.1)$ \\
AK FPB & 0 & $17(18.3)$ \\
AK FPB plus tibial artery & 0 & $23(24.7)$ \\
BK FPB & 0 & $12(12.9)$ \\
BK FPB plus tibial artery & & 0.238 \\
\hline
\end{tabular}

AK, above the knee; BK, below the knee; DPA, dorsalis pedis artery; EVT, endovascular therapy; FPB, femoropopliteal bypass; PTA, posterior tibial artery; TASC, intersociety consensus for the management of peripheral arterial disease. 
TABLE 3: Study endpoints associated with treatment methods.

\begin{tabular}{|c|c|c|c|}
\hline Parameters & EVT group $(n=94)$ & Bypass group $(n=93)$ & $P$ value \\
\hline Successful intervention at one month & $93(100)$ & $94(100)$ & \\
\hline Direct revascularization & $43(45.7)$ & $47(50.5)$ & 0.512 \\
\hline \multicolumn{4}{|l|}{ Postintervention ABI } \\
\hline One month & $0.92 \pm 0.1$ & $0.95 \pm 0.16$ & 0.113 \\
\hline 36 months & $0.53 \pm 0.16$ & $0.72 \pm 0.14$ & $<0.001$ \\
\hline \multicolumn{4}{|l|}{ Primary patency } \\
\hline 12 months & $70(74.5)$ & $76(81.7)$ & 0.231 \\
\hline 24 months & $52(55.3)$ & $62(66.7)$ & 0.112 \\
\hline 36 months & $37(39.4)$ & $55(59.1)$ & 0.007 \\
\hline \multicolumn{4}{|l|}{ Secondary patency } \\
\hline 24 months & $74(78.7)$ & $79(84.9)$ & 0.27 \\
\hline 36 months & $61(64.9)$ & $73(78.5)$ & 0.039 \\
\hline Male & $25(26.6)$ & $31(33.3)$ & 0.315 \\
\hline AFSR at 36 months & $64(68.1)$ & $71(76.3)$ & 0.208 \\
\hline LEA at 36 months & $32(34.0)$ & $29(31.2)$ & 0.954 \\
\hline Major & $8(8.5)$ & $8(8.6)$ & \\
\hline Minor & $23(24.5)$ & $21(22.6)$ & \\
\hline 24-month mortality & $15(16.0)$ & $9(9.7)$ & 0.199 \\
\hline 36-month mortality & $27(28.7)$ & $18(19.4)$ & 0.134 \\
\hline 12-month CAD events & $15(16.0)$ & $17(18.3)$ & 0.673 \\
\hline 24-month CAD events & $28(29.8)$ & $26(28.0)$ & 0.782 \\
\hline 36-month CAD events & $30(31.9)$ & $29(31.2)$ & 0.914 \\
\hline 24-month CVA events & $5(5.3)$ & $10(10.8)$ & 0.171 \\
\hline 36-month CVA events & $10(10.6)$ & $11(11.8)$ & 0.797 \\
\hline \multicolumn{4}{|l|}{ Concomitant medications } \\
\hline \multicolumn{4}{|l|}{ Cilostazol treatment } \\
\hline$\leq 3$ months & $31(33.0)$ & $23(24.7)$ & 0.213 \\
\hline$>3$ months & $63(67.0)$ & $70(75.3)$ & \\
\hline Statin treatment & $32(34.0)$ & $42(45.2)$ & 0.120 \\
\hline Antiplatelet therapy & $93(1001)$ & $94(100)$ & 1.000 \\
\hline
\end{tabular}

$\mathrm{ABI}$, ankle brachial index; AFSR, amputation-free survival rate; CAD, coronary artery disease; CVA, cerebrovascular accident; EVT, endovascular therapy; LEA, lower-extremity amputation; MALE, major adverse limb event.
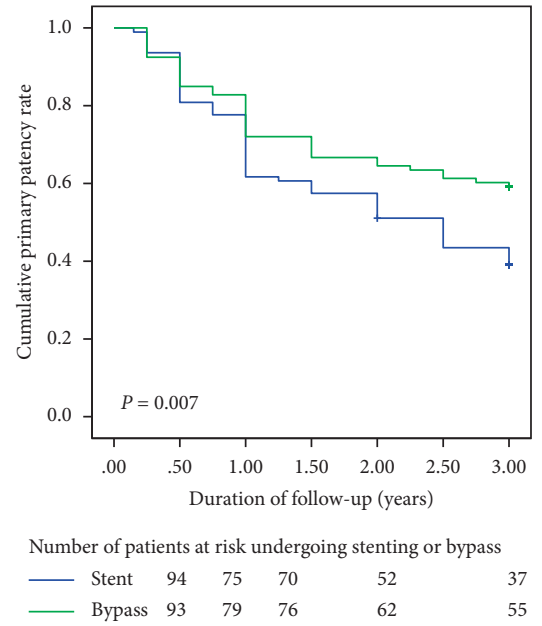

(a)

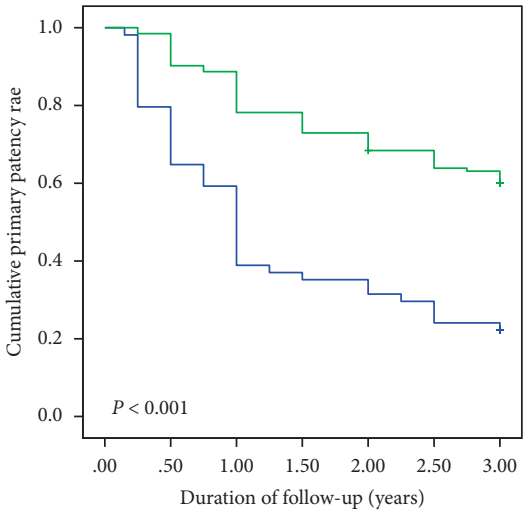

Number of patients at risk with cilostazol

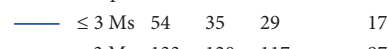

(b)

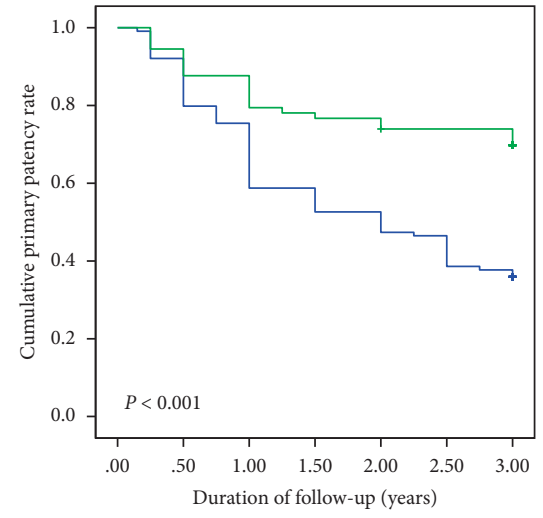

Number of patients at risk with a statin

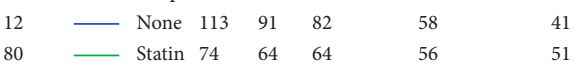

(c)

Figure 1: (a) Patients who underwent bypass surgery had a better patency rate than patients who received stenting treatment (log rank test, $P=0.007)$. (b) Patients who received cilostazol treatment for more than 3 months had a patency rate than patients who received treatment for fewer than 3 months (log rank test, $P<0.001$ ). (c) Patients receiving statin treatment had a patency rate than those who did not receive treatment (log rank test, $P<0.001)$. 

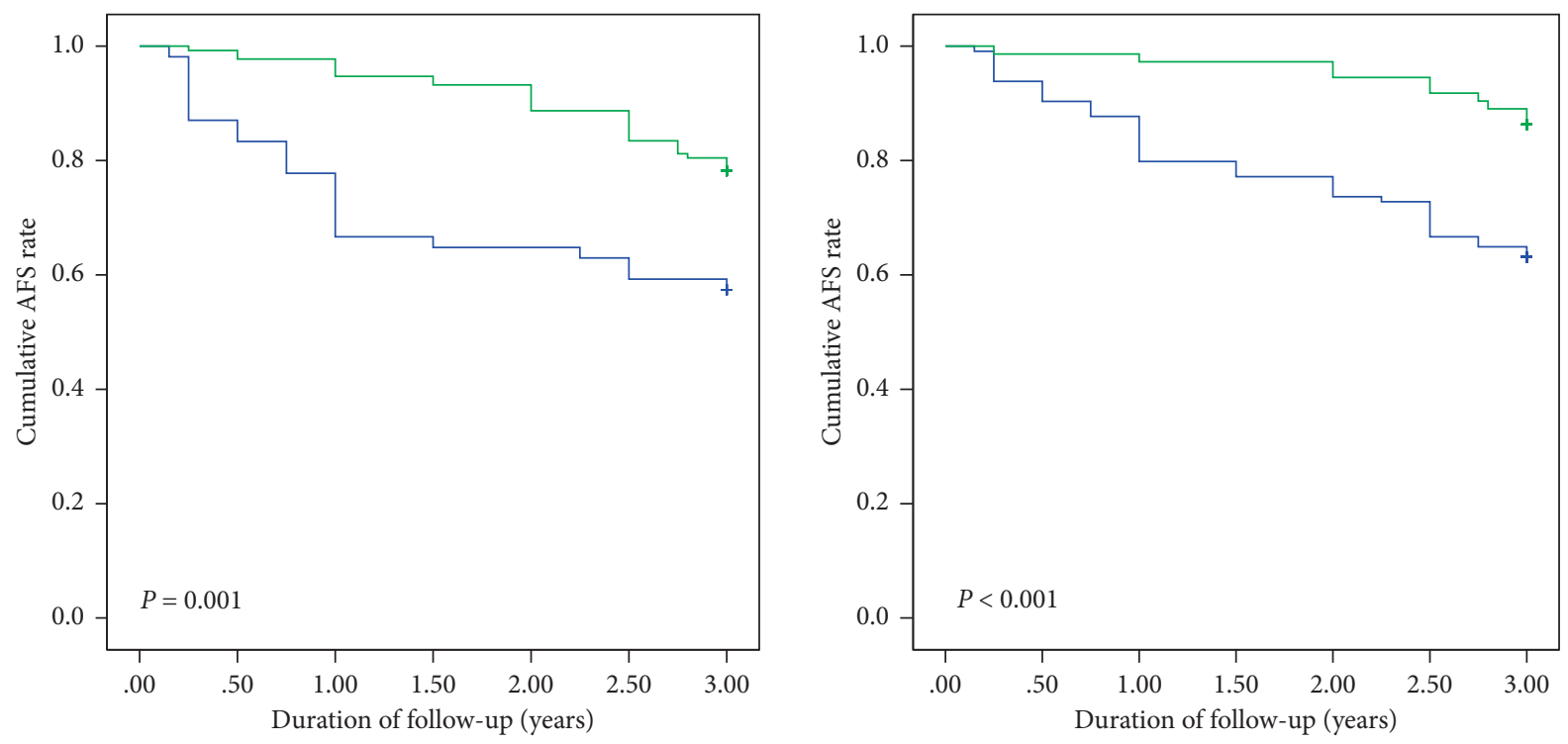

Number of patients at risk with cilostazol

$\begin{array}{llllll}\leq 3 \mathrm{Ms} & 54 & 45 & 36 & 35 & 31 \\ ->3 \mathrm{Ms} & 133 & 130 & 126 & 118 & 104\end{array}$

Number of patients at risk with a statin

$\begin{array}{lllll}\text { - None } 113 & 102 & 90 & 83 & 71 \\ \text { - Statin } 74 & 73 & 72 & 70 & 64\end{array}$

(a)

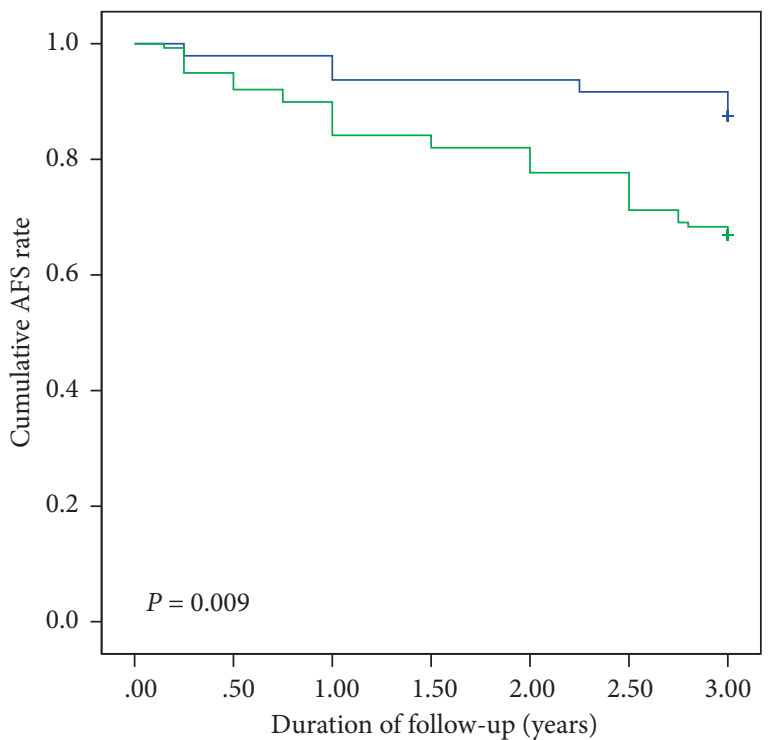

(b)

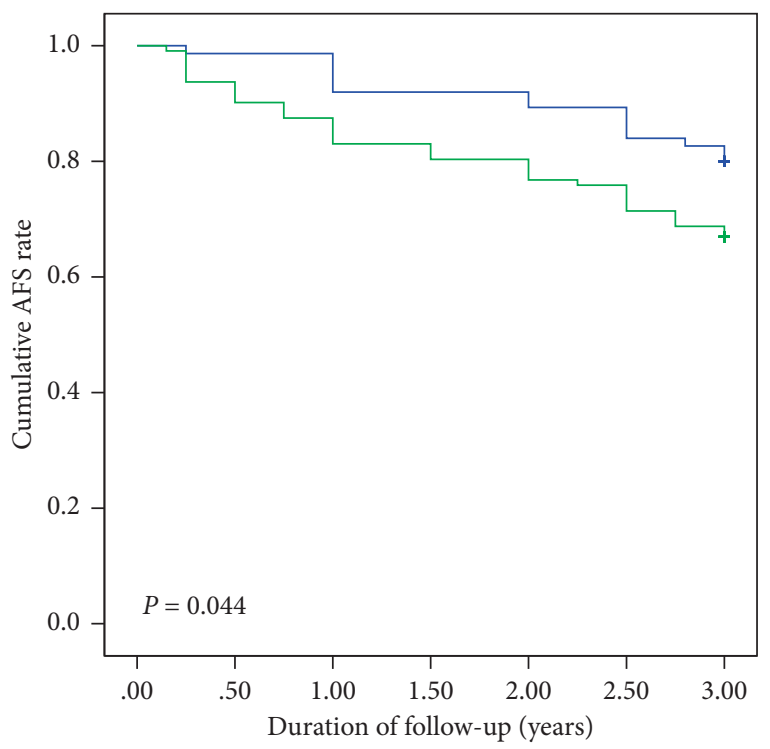

Number of patients at risk

$\begin{array}{llllll}\text { — Rutherford } 2 / 3 & 48 & 47 & 45 & 45 & 42 \\ \text { Rutherford } 4 & 139 & 128 & 117 & 108 & 93\end{array}$

(c)
Number of patients at risk

$\begin{array}{llllll}\text { - None } & 75 & 74 & 69 & 67 & 60 \\ \text { DM } & 112 & 101 & 93 & 86 & 75\end{array}$

(d)

FIgURe 2: (a) Patients who received cilostazol treatment for more than 3 months had a better 3-year amputation-free survival (AFS) rate than patients who received treatment for fewer than 3 months (log rank test, $P=0.001$ ). (b) Patients receiving statin treatment had a superior 3-year AFS rate to those who did not receive treatment $(\log$ rank test, $P<0.001)$. (c) Patients who presented with TASC D peripheral artery disease (PAD) had an inferior 3-year AFS rate than patients who had TASC B plus C PAD (log rank test, $P=0.009)$. (d) Patients with diabetes mellitus had a poorer 3-year AFS rate than patients without DM ( $\log$ rank test, $P=0.044$ ).

(D) patients with diabetes mellitus had a poorer 3-year AFS than patients without (log rank test, $P=0.044$ ).

The 3-year AFS was $68.1 \%$ (64) and $76.3 \%(71)$ in the ETV and bypass groups, respectively $(P=0.208)$, while the 24- and 36-month OS rates were similar in the two groups
$(P=0.199$ and $P=0.134)$. Regarding new CAD and CVA events, there were no significant differences between the ETV and bypass groups at 12, 24, and 36 months. In total, 31 patients died during the 36-month follow-up period, 17 due to cardiogenic shock related to cardiovascular disease and 7 
due to associated foot sepsis; three uremic patients died due to complications of major surgery, and the remaining 4 expired due to sepsis related to pneumonia.

3.3. Assessment of Primary Patency. In the weighted univariate Cox regression analysis of the 36-month primary patency, the significant factors were long-term cilostazol usage, statin treatment, insulin use, diabetic neuropathy, intervention (bypass/stenting), and DR. The results of multivariate Cox regression analysis showed that three main factors were independently associated with primary patency for 36 months: cilostazol treatment for more than 3 months (HR: $0.46,95 \%$ CI: $0.3-0.72, P=0.001$ ), statin treatment (HR: 0.54, 95\% CI: 0.33-0.9, $P=0.0179$ ), and DR (HR: 0.47, 95\% CI: $0.29-0.74, P=0.001$ ) (Table 4 ).

Furthermore, in the subgroup multivariate Cox regression analysis, cilostazol treatment (HR: 0.46, 95\% CI: $0.26-0.82, P=0.009$ ) and DR (HR: $0.43,95 \%$ CI: $0.22-0.81$, $P=0.009)$ were independent factors associated with the 36 month primary patency in the EVT group, whereas the only main factor in the bypass group was cilostazol treatment (HR: 0.33, 95\% CI: 0.15-0.75, $P=0.008$ ) (Table 5).

\section{Discussion}

Restenosis and intimal hyperplasia are the main problems that occur after endovascular revascularization with percutaneous transluminal angioplasty or stenting. Despite the popularity and advancement of endovascular revascularization for CLI, improving the clinical outcome and preservation of limb function remain challenging following revascularization in long femoropopliteal lesions.

In this study, almost $60 \%$ of the patients had diabetes, $38 \%$ had CAD, 27.4\% had hyperlipidemia, $82.4 \%$ had hypertension, and more than $90 \%$ of patients met the criteria for CLI. Most of the patients had at least two levels of complicated arterial occlusion, mainly involving femoropopliteal and infrapopliteal lesions, and partly aortoiliac lesions. In comparison with published literature regarding self-expanding polytetrafluoroethylene (ePTFE)-covered stents (Viabahn; W. L. Gore and Associates, Inc., Flagstaff, AZ, USA) and bare metal stents (BMS) [15-17], which reported the percentage of CTO lesions to be $56-70 \%$, with $35-44 \%$ of patients having diabetes, $26-28 \%$ experiencing hyperlipidemia, 22-36\% with CAD, and 14-19\% experiencing tissue loss, the percentage of patients with comorbidities was higher in this study.

Numerous studies have demonstrated that a greater number of comorbid conditions and risk factors are associated with poor durability of patency, high risk of mortality, and failure of limb salvage [18-21]. In comparison with these aforementioned studies, our patient group was of a more complex composition, but the 2-year major amputation rate (7.3\% vs. $8.0 \%)$, 2-year primary patency rate $(63.6 \%$ vs. $56.4 \%)$, and 2 -year secondary patency rate were comparable $(85.5 \%$ vs. $80.9 \%)$. The main reason for the inferior 2 -year primary patency rate was that the stenting instruments used in this study included BMS and Viabahn for femoropopliteal lesions, combined with balloon angioplasty for infrapopliteal lesions; Viabahn was not available in our institution prior to October 2011, and therefore BMS was the only device being used before that time. Numerous recent trials have demonstrated that BMS provides an efficient treatment for short lesions but is subject to neointimal hyperplasia and carries risks of in-stent restenosis (ISR) and stent fracture when used in long lesions, while the covered stent (Viabahn) offers more ideal outcomes in long stenotic or occlusive femoropopliteal lesions (TASC C and D lesions) and satisfactory prevention of neointimal growth, but with frequent occurrence of edge stenosis with graft thrombosis [15, 22-26].

The results of this study showed the 3-year primary and secondary patency rates of the bypass group to be $65.6 \%$ and $81.7 \%$, as compared with several bypass studies reporting primary patency rates of $57.2-76.2 \%$ and $62.6-81.8 \%$ [27]. This study did not present a notable 3 -year primary patency rate, and there was a comparable 3-year secondary patency rate because this study included patients with long, complex, and multilevel arterial lesions.

The Bypass Versus Angioplasty in Severe Ischemia of the Leg (BASIL) study demonstrated that the surgical group had a lower rate of repeated revascularization and no differences in major amputation or mortality for more than 5 years as compared with the endovascular group were noted, despite the higher risks of myocardial infarction, wound infection, and pulmonary complications [28]. Our study presented comparable 1- and 2-year primary patency rates and no differences in mortality, amputation, or cardiovascular events at the 3-year follow-up point, but the bypass group had better 3-year primary and secondary patency rates. Our finding that bypass surgery may provide a more durable patency for long SFA lesions as compared with EVT was partly concordant with the results of the BASIL study [28]. Endovascular devices and techniques have been greatly improved since the BASIL trial, and there are more strategies for maintenance of graft patency and improvement of limb salvage with endovascular intervention [29-33]. To date, endovascular revascularization has been advocated as the first option for CLI treatment, but long and complicated SFA lesions are subject to flexion, compression, and torsion close to the popliteal area, which leads to poorer durability of BMS and graft stenting, whereas surgical revascularization provides a straight-line flow into the foot, promotes wound healing, and limits the need for amputation. Accordingly, surgical bypass may be an alternative option for the treatment of long lesions in advanced PAD. Taking these results together, the optimal cure strategy (bypass surgery versus endovascular revascularization) demands a comprehensive understanding of anatomic configurations, patient conditions and preferences, surgeon experience and skill, and a multidisciplinary approach to promote a better clinical outcome and quality of life.

Evidence that pharmacologic therapies prevent restenosis or stent thrombosis after bypass surgery or endovascular therapy is sparse and often inferred from studies of coronary artery interventions regarding antiplatelet therapy. The ACC/AHA guidelines for PAD management recommend that cilostazol treatment is effective in improving 
TABLE 4: Cox regression analysis of primary patency.

\begin{tabular}{|c|c|c|c|c|}
\hline \multirow{2}{*}{ Variable } & \multicolumn{2}{|c|}{ Univariate } & \multicolumn{2}{|c|}{${ }^{\#}$ Multivariate } \\
\hline & $\mathrm{HR}^{*}\left(95 \% \mathrm{CI}^{\dagger}\right)$ & $P$ value & ATE & $P$ value \\
\hline Age (years) & $1.02(0.99-1.04)$ & 0.208 & & \\
\hline Gender (F/M) & $1.01(0.7-1.77)$ & 0.662 & & \\
\hline BMI & $0.99(0.94-1.04)$ & 0.59 & & \\
\hline Smoking (yes/no) & $1.36(0.89-2.08)$ & 0.155 & & \\
\hline Hyperlipidemia (yes/no) & $0.97(0.55-1.65)$ & 0.902 & & \\
\hline CAD (yes/no) & $0.95(0.63-1.42)$ & 0.798 & & \\
\hline Hypertension (yes/no) & $1.06(0.66-1.72)$ & 0.799 & & \\
\hline DM (yes/no) & $1.26(0.81-1.96)$ & 0.306 & & \\
\hline ESRD (yes/no) & $1.06(0.67-1.69)$ & 0.805 & & \\
\hline Rutherford classification (V plus VI/III plus IV) & $1.46(0.87-2.46)$ & 0.153 & & \\
\hline hsCRP (mg/L) & $1.03(0.99-1.67)$ & 0.1 & $1.02(0.98-1.0)$ & 0.399 \\
\hline Diabetic neuropathy & $2.03(1.34-3.08)$ & 0.001 & $0.94(0.5-1.74)$ & 0.824 \\
\hline \multicolumn{5}{|l|}{ Ischemia severity } \\
\hline $\mathrm{ABI}$ & $1.26(0.78-2.02)$ & 0.341 & & \\
\hline Statin treatment (yes/no) & $0.38(0.24-0.62)$ & $<0.001$ & $0.54(0.33-0.9)$ & 0.017 \\
\hline Cilostazol treatment $(>3 \mathrm{Ms} / \leq 3 \mathrm{Ms})$ & $0.33(0.22-0.51)$ & $<0.001$ & $0.46(0.3-0.72)$ & 0.001 \\
\hline Insulin alone (yes/no) & $2.12(1.38-3.25)$ & 0.001 & $1.6(0.91-2.81)$ & 0.106 \\
\hline Involved leg (L/R) & $1.18(0.82-1.7)$ & 0.364 & & \\
\hline Intervention (bypass/stenting) & $0.61(0.41-0.92)$ & 0.019 & $0.8(0.52-1.22)$ & 0.292 \\
\hline $\mathrm{DR} / \mathrm{IR}$ & $0.34(0.22-0.53)$ & $<0.001$ & $0.47(0.29-0.74)$ & 0.001 \\
\hline
\end{tabular}

${ }^{*}$ Multivariate, weighted Cox regression with average treatment effect (ATE), ${ }^{*} \mathrm{HR}$, hazard ratio; ${ }^{\dagger} \mathrm{CI}$, confidence interval. ABI, ankle brachial index; BMI, body mass index; CAD, coronary artery disease; DM, diabetes mellitus; ESRD, end-stage renal disease; DR, direct revascularization; hsCRP: high-sensitivity Creactive protein; HAlc: hemoglobin Alc; IR, indirect revascularization.

TABLe 5: Multivariate Cox regression analysis of primary patency in the two groups.

\begin{tabular}{|c|c|c|c|c|}
\hline \multirow{2}{*}{ Variable } & \multicolumn{2}{|c|}{ Multivariate (EVT group) } & \multicolumn{2}{|c|}{ Multivariate (bypass group) } \\
\hline & $\mathrm{HR}^{*}\left(95 \% \mathrm{CI}^{\dagger}\right)$ & $P$ value & $\mathrm{HR}^{*}\left(95 \% \mathrm{CI}^{\dagger}\right)$ & $P$ value \\
\hline hsCRP (mg/L) & $1.02(0.54-1.92)$ & 0.96 & $1.96(0.77-5.0)$ & 0.157 \\
\hline Neuropathy & $0.83(0.38-1.82)$ & 0.648 & $0.89(0.29-2.71)$ & 0.832 \\
\hline Statin treatment (yes/no) & $0.74(0.38-1.45)$ & 0.381 & $0.59(0.25-1.42)$ & 0.241 \\
\hline Cilostazol treatment $(>3 \mathrm{Ms} / \leq 3 \mathrm{Ms})$ & $0.46(0.26-0.82)$ & 0.009 & $0.33(0.15-0.75)$ & 0.008 \\
\hline Insulin alone (yes/no) & $0.98(0.46-2.09)$ & 0.966 & $2.72(0.94-7.87)$ & 0.065 \\
\hline DR/IR & $0.43(0.22-0.81)$ & 0.009 & $0.5(0.2-1.23)$ & 0.132 \\
\hline
\end{tabular}

${ }^{*} \mathrm{HR}$, hazard ratio; ${ }^{\dagger} \mathrm{CI}$, confidence interval; DR, direct revascularization; EVT, endovascular therapy; hsCRP: high-sensitivity C-reactive protein; HA1c: hemoglobin Alc; IR, indirect revascularization.

intermittent claudication and increasing walking distance [34]. Clinical studies of the benefit of cilostazol treatment for improvement of patency and limb salvage are rare, and most trials suggest that cilostazol may reduce the ISR in patients with coronary intervention and those with femoropopliteal lesions [35-39].

Cilostazol is an antiplatelet drug with multiple effects, including inhibition of platelet aggregation and proliferation of smooth muscle cells, and then promotion of vasodilation and increased peripheral blood flow [40-42]. Accordingly, this preliminary study demonstrated that long-term administration of cilostazol was a significant predictor of 36month primary patency and AFS after open surgical or endovascular revascularization. However, this study provided no direct evidence that cilostazol treatment led to improvements in wound healing or limb salvage. Further research is needed in this field.

This study also assessed whether the use of statins is associated with primary patency of target lesions. Of the 187 patients, $74(39.6 \%)$ received postoperative treatment with statins to control total cholesterol and LDL levels. The results demonstrated that the patients under long-term statin therapy experienced great benefits in terms of the 3-year patency of target lesions and increased AFS. This result was somewhat in line with the report of the CRITISCH registry, which indicated that the use of statins in patients with CLI is associated with a better AFS and a lower LEA and mortality rate [43]. Further studies focusing on the effects of statin treatment should be carried out to clarify this issue.

Several trials have demonstrated that the outcomes in terms of wound healing and limb preservation after IR in the presence of collaterals are similar to the outcomes after DR $[8-10,44]$. Our multivariate Cox regression analyses of patency revealed that DR may provide a straight-line flow to the foot and improve the primary patency at 36 months in the bypass and EVT groups. Especially, DR may be an independent predictor of 3-year primary patency after endovascular stenting. Further research may be needed in this field. 
There were some limitations to our study. The first was that this study was a single-center, retrospective analysis of a prospectively-maintained database that did not have sufficient functionality for extensive statistical comparisons. Second, this was not an analysis of single femoropopliteal lesions or TASC classification, which may have led to some differences being derived from selection bias. To reduce bias, weighted multivariate Cox regression analysis using a propensity score-based approach was used to assess the associations of factors based on TASC disease severity and location with primary patency. Third, the time interval during which the patients were enrolled was long, and changes in the techniques and devices employed could have influenced the results.

\section{Conclusions}

Our study did not show any significant difference in outcome between endovascular stenting based on the angiosome concept and bypass surgery for the treatment of long, advanced femoropopliteal lesions after a short follow-up period, whereas cilostazol therapy for more than 3 months, aggressive treatment of dyslipidemia, and surgical revascularization were associated with higher primary patency. A large-scale, prospective, randomized study should be conducted for further exploration.

\section{Data Availability}

The data used to support the findings of this study are available from the corresponding author upon request after the author gets approval from the ethics committee.

\section{Conflicts of Interest}

The author declares no conflicts of interest.

\section{References}

[1] B. T. Rodrigues, V. N. Vangaveti, and U. H. Malabu, "Prevalence and risk factors for diabetic lower limb amputation: a clinic-based case control study," Journal of Diabetes Research, vol. 2016, Article ID 5941957, 7 pages, 2016.

[2] M. I. Spreen, H. Gremmels, M. Teraa et al., "Diabetes is associated with decreased limb survival in patients with critical limb ischemia: pooled data from two randomized controlled trials," Diabetes Care, vol. 39, no. 11, pp. 2058-2064, 2016.

[3] L. Norgren, W. R. Hiatt, J. A. Dormandy et al., "Inter-society consensus for the management of peripheral arterial disease (TASC II)," European Journal of Vascular and Endovascular Surgery, vol. 33, pp. S1-S75, 2007.

[4] M. S. Hong, A. W. Beck, and P. R. Nelson, "Emerging national trends in the management and outcomes of lower extremity peripheral arterial disease," Annals of Vascular Surgery, vol. 25, no. 1, pp. 44-54, 2011.

[5] W. S. Jones, X. Mi, L. G. Qualls et al., "Trends in settings for peripheral vascular intervention and the effect of changes in the outpatient prospective payment system," Journal of the American College of Cardiology, vol. 65, no. 9, pp. 920-927, 2015.
[6] J. A. Dormandy and R. B. Rutherford, "Management of peripheral arterial disease (PAD)," Journal of Vascular Surgery, vol. 31, no. 1, pp. S1-S296, 2000.

[7] P. Brosi, F. Dick, D. D. Do, J. Schmidli, I. Baumgartner, and N. Diehm, "Revascularization for chronic critical lower limb ischemia in octogenarians is worthwhile," Journal of Vascular Surgery, vol. 46, no. 6, pp. 1198-1207, 2007.

[8] O. Iida, Y. Soga, K. Hirano et al., "Long-term results of direct and indirect endovascular revascularization based on the angiosome concept in patients with critical limb ischemia presenting with isolated below-the-knee lesions," Journal of Vascular Surgery, vol. 55, no. 2, pp. 363-370, 2012.

[9] H. Jongsma, J. A. Bekken, G. P. Akkersdijk, S. E. Hoeks, H. J. Verhagen, and B. Fioole, "Angiosome-directed revascularization in patients with critical limb ischemia," Journal of Vascular Surgery, vol. 65, no. 4, pp. 1208-1219, 2017.

[10] A. Lejay, Y. Georg, E. Tartaglia et al., "Long-term outcomes of direct and indirect below-the-knee open revascularization based on the angiosome concept in diabetic patients with critical limb ischemia," Annals of Vascular Surgery, vol. 28, no. 4, pp. 983-989, 2014.

[11] M. R. Patel, M. S. Conte, D. E. Cutlip et al., "Evaluation and treatment of patients with lower extremity peripheral artery disease," Journal of the American College of Cardiology, vol. 65, no. 9, pp. 931-941, 2015.

[12] J. L. Mills, M. S. Conte, D. G. Armstrong et al., "The society for vascular surgery lower extremity threatened limb classification system: risk stratification based on wound, ischemia, and foot infection (WIfI)," Journal of Vascular Surgery, vol. 59, no. 1, pp. 220-234, 2014.

[13] T. Stürmer, R. Wyss, R. J. Glynn, and M. A. Brookhart, "Propensity scores for confounder adjustment when assessing the effects of medical interventions using nonexperimental study designs," Journal of Internal Medicine, vol. 275, no. 6, pp. 570-580, 2014.

[14] Y. Zhang, U. G. Gerdtham, H. Rydell, and J. Jarl, "Quantifying the treatment effect of kidney transplantation relative to dialysis on survival time: new results based on propensity score weighting and longitudinal observational data from Sweden," International Journal of Environmental Research and Public Health, vol. 17, no. 19, 2020.

[15] J. Lammer, T. Zeller, K. A. Hausegger et al., "Heparin-bonded covered stents versus bare-metal stents for complex femoropopliteal artery lesions: the randomized VIASTAR trial (Viabahn endoprosthesis with PROPATEN bioactive surface [VIA] versus bare nitinol stent in the treatment of long lesions in superficial femoral artery occlusive disease)," Journal of the American College of Cardiology, vol. 62, no. 15, pp. 1320-1327, 2013.

[16] P. J. Geraghty, M. W. Mewissen, M. R. Jaff, and G. M. Ansel, "Three-year results of the VIBRANT trial of VIABAHN endoprosthesis versus bare nitinol stent implantation for complex superficial femoral artery occlusive disease," Journal of Vascular Surgery, vol. 58, no. 2, pp. 386-395, 2013.

[17] J. Lammer, T. Zeller, K. A. Hausegger et al., "Sustained benefit at 2 years for covered stents versus bare-metal stents in long SFA lesions: the VIASTAR trial," CardioVascular and Interventional Radiology, vol. 38, no. 1, pp. 25-32, 2015.

[18] O. Iida, M. Uematsu, Y. Soga et al., "Timing of the restenosis following nitinol stenting in the superficial femoral artery and the factors associated with early and late restenoses," Catheterization and Cardiovascular Interventions, vol. 78, no. 4, pp. 611-617, 2011. 
[19] E. Van Belle, S. Nikol, L. Norgren et al., "Insights on the role of diabetes and geographic variation in patients with criticial limb ischaemia," European Journal of Vascular and Endovascular Surgery, vol. 42, no. 3, pp. 365-373, 2011.

[20] J. Belch, W. R. Hiatt, I. Baumgartner et al., "Effect of fibroblast growth factor NV1FGF on amputation and death: a randomised placebo-controlled trial of gene therapy in critical limb ischaemia," The Lancet, vol. 377, no. 9781, pp. 1929-1937, 2011.

[21] A. J. Meltzer, G. Evangelisti, A. R. Graham et al., "Determinants of outcome after endovascular therapy for critical limb ischemia with tissue loss," Annals of Vascular Surgery, vol. 28, no. 1, pp. 144-151, 2014.

[22] P. Kougias, A. Chen, C. Cagiannos, C. F. Bechara, T. T. Huynh, and P. H. Lin, "Subintimal placement of covered stent versus subintimal balloon angioplasty in the treatment of long-segment superficial femoral artery occlusion," The American Journal of Surgery, vol. 198, no. 5, pp. 645-649, 2009.

[23] P. Dick, H. Wallner, S. Sabeti et al., "Balloon angioplasty versus stenting with nitinol stents in intermediate length superficial femoral artery lesions," Catheterization and Cardiovascular Interventions, vol. 74, no. 7, pp. 1090-1095, 2009.

[24] M. Schillinger, S. Sabeti, C. Loewe et al., "Balloon angioplasty versus implantation of nitinol stents in the superficial femoral artery," New England Journal of Medicine, vol. 354, no. 18, pp. 1879-1888, 2006.

[25] J. R. Laird, B. T. Katzen, D. Scheinert et al., "Nitinol stent implantation versus balloon angioplasty for lesions in the superficial femoral artery and proximal popliteal artery," Circulation: Cardiovascular Interventions, vol. 3, no. 3, pp. 267-276, 2010.

[26] D. Scheinert, S. Scheinert, J. Sax et al., "Prevalence and clinical impact of stent fractures after femoropopliteal stenting," Journal of the American College of Cardiology, vol. 45, no. 2, pp. 312-315, 2005.

[27] C. E. Pereira, M. Albers, M. Romiti, F. C. Brochado-Neto, and C. A. B. Pereira, "Meta-analysis of femoropopliteal bypass grafts for lower extremity arterial insufficiency," Journal of Vascular Surgery, vol. 44, no. 3, pp. 510-517, 2006.

[28] D. J. Adam, J. D. Beard, T. Cleveland et al., "Bypass versus angioplasty in severe ischaemia of the leg (BASIL): multicentre, randomised controlled trial," Lancet (London, England), vol. 366, no. 9501, pp. 1925-1934, 2005.

[29] J. A. Michaels, "Choice of material for above-knee femoropopliteal bypass graft," British Journal of Surgery, vol. 76, no. 1, pp. 7-14, 1989.

[30] J. Berglund, M. Björck, J. Elfström, and SWEDVASC FemoroPopliteal Study Group, "Long-term results of above knee femoro-popliteal bypass depend on indication for surgery and graft-material," European Journal of Vascular and Endovascular Surgery, vol. 29, no. 4, pp. 412-418, 2005.

[31] B. S. Maini, R. K. Orr, P. O'Mara, and T. Hendershott, "Outcomes and resource utilization in a managed care setting for lower extremity vein bypass grafts," The American Journal of Surgery, vol. 172, no. 2, pp. 113-117, 1996.

[32] S. Raptis and J. H. Miller, "Influence of a vein cuff on polytetrafluoroethylene grafts for primary femoropopliteal bypass," British Journal of Surgery, vol. 82, no. 4, pp. 487-491, 1995.

[33] M. D. Gerhard-Herman, H. L. Gornik, C. Barrett et al., "2016 AHA/ACC guideline on the management of patients with lower extremity peripheral artery disease: a report of the American College of Cardiology/American Heart Association
Task Force on clinical practice guidelines," Circulation, vol. 135, no. 12, pp. e726-e779, 2017.

[34] M. F. Zuliani Mauro, J. A. Mangione, J. R. Costa Jr. et al., "Randomized angiographic and intravascular ultrasound comparison of dual-antiplatelet therapy vs. triple-antiplatelet therapy to reduce neointimal tissue proliferation in diabetic patients," Journal of Invasive Cardiology, vol. 29, no. 3, pp. 76-81, 2017.

[35] O. Iida, S. Nanto, M. Uematsu, T. Morozumi, M. Kitakaze, and S. Nagata, "Cilostazol reduces restenosis after endovascular therapy in patients with femoropopliteal lesions," Journal of Vascular Surgery, vol. 48, no. 1, pp. 144-149, 2008.

[36] Y. Soga, H. Yokoi, T. Kawasaki et al., "Efficacy of cilostazol after endovascular therapy for femoropopliteal artery disease in patients with intermittent claudication," Journal of the American College of Cardiology, vol. 53, no. 1, pp. 48-53, 2009.

[37] O. Iida, H. Yokoi, Y. Soga et al., "Cilostazol reduces angiographic restenosis after endovascular therapy for femoropopliteal lesions in the sufficient treatment of peripheral intervention by cilostazol study," Circulation, vol. 127, no. 23, pp. 2307-2315, 2013.

[38] S. Bangalore, A. Singh, B. Toklu et al., "Efficacy of cilostazol on platelet reactivity and cardiovascular outcomes in patients undergoing percutaneous coronary intervention: insights from a meta-analysis of randomised trials," Open Heart, vol. 1, no. 1, Article ID e000068, 2014.

[39] C.-Y. Lee, T.-C. Wu, and S.-J. Lin, "Effects of postoperative percutaneous coronary intervention, pharmacologic treatment, and predisposing factors on clinical outcomes in patients with and without type 2 diabetes along with critical limb ischemia," Clinical Therapeutics, vol. 43, no. 1, pp. 195-210, 2021.

[40] W.-J. Chen, Y.-H. Chen, K.-H. Lin, C. Hsuan Ting, and Y.-H. Yeh, "Cilostazol promotes vascular smooth muscles cell differentiation through the cAMP response element-binding protein-dependent pathway," Arteriosclerosis, Thrombosis, and Vascular Biology, vol. 31, no. 9, pp. 2106-2113, 2011.

[41] S. Hayashi, R. Morishita, H. Matsushita et al., "Cyclic AMP inhibited proliferation of human aortic vascular smooth muscle cells, accompanied by induction of p53 and p21," Hypertension, vol. 35, no. 1, pp. 237-243, 2000.

[42] F. Sanada, Y. Kanbara, Y. Taniyama et al., "Induction of angiogenesis by a type III phosphodiesterase inhibitor, cilostazol, through activation of peroxisome proliferator-activated receptor- $\gamma$ and cAMP pathways in vascular cells," Arteriosclerosis, Thrombosis, and Vascular Biology, vol. 36, no. 3, pp. 545-552, 2016.

[43] K. Stavroulakis, M. Borowski, G. Torsello et al., "Association between statin therapy and amputation-free survival in patients with critical limb ischemia in the CRITISCH registry," Journal of Vascular Surgery, vol. 66, no. 5, pp. 1534-1542, 2017.

[44] M. D. Gerhard-Herman, H. L. Gornik, C. Barrett et al., "2016 AHA/ACC guideline on the management of patients with lower extremity peripheral artery disease: executive summary: a report of the American College of Cardiology/American Heart Association Task Force on clinical practice guidelines," Journal of the American College of Cardiology, vol. 69, no. 11, pp. 1465-1508, 2017. 